\title{
Introduction
}

\section{Mental Dysfunction in Parkinson's Disease}

For a long time, Parkinson's disease was considered almost exclusively a motor disorder. With the development of efficacious therapies against the motor manifestations, however, the non-motor aspects have surfaced from the background to become an important topic of research. Their relevance encompasses the understanding of the disease process and its progression, their importance in establishing the diagnosis, and, of course addressing the needs of the patients.

Significant advances these fields have been made over the past two decades include the recognition of sensory abnormalities, particularly olfactory and visual dysfunctions, and sleep impairment including REM-sleep behavior disorder - all of which can be typically observed years before the emergence of the first motor symptoms, as well as cognitive and behavioral changes during the evolution of the disease.

These developments have been followed in a series of congresses, which started in 1993 and gradually attracted attention and popularity. The present volume contains several of the more important contributions presented at last year's congress held in Seoul, Korea.

The next congress in this series, The 10th International Congress on Non-Motor Dysfunctions in Parkinson's Disease and Related Disorders (http://www.kenes.com/nmdpd2014/), will take place in Nice, France, December $2-8,2014$, and will surely add even more new information on the topics, which are so important to all clinicians and basic scientists in the field of movement disorders.

Professor Emeritus Amos D. Korczyn

Department of Neurology

Tel Aviv University

Ramat Aviv 69978, Israel

E-mail: amoskor@tau.ac.il 